\title{
The Catalogue of Martian Mössbauer Spectra
}

\author{
A. Maliszewski, J. Galązka-Friedman, K. Szlachta and M. Urbanski
}

Faculty of Physics, Warsaw University of Technology, 00-662 Warszawa, Koszykowa 75, Poland

The suggestion of sending a Mössbauer spectrometer to the surface of Mars was elaborated in 1988 year. In 1989 realization of this project was taken by scientists from Western Europe and USSR. Finally a Mössbauer spectrometer adapted to measurements on the surface of Mars was constructed by an international team under the guidance of G. Klingelhöfer. Mössbauer data from Mars are available for open public access on a web site of Planetary Data System. Based on these data the catalogue of Martian Mössbauer spectra was prepared and located on the web site of one of the author (JGF). The aim of the construction of this catalogue was to create the possibility to compare quickly spectra from different places on the Martian surface.

PACS: $91.25 . \mathrm{F}-$

\section{Introduction}

The suggestion of sending a Mössbauer spectrometer to the surface of Mars was elaborated in 1988 year [1]. At the beginning three Polish teams were working on this subject and the final version of this project was prepared by scientists from the Cosmic Research Center of Polish Academy of Sciences in Warsaw [2].

In 1989 the realization of this project was taken by scientists from Western Europe and the USSR. Finally a Mössbauer spectrometer adapted for measurements on the surface of Mars was constructed by an international team under the guidance of G. Klingelhöfer [3]. Miniaturized Mössbauer spectrometers were located on one European and two NASA rockets. These rockets were sent to Mars in the middle of 2003. The European Rover did not start to work on the surface of Mars but the American mission ended as a success.

Mössbauer data from Mars are available for open public access on a web site of Planetary Data System. Based on these data the catalogue of Martian Mössbauer spectra was prepared and located on the web site of one of the authors (JGF).

\section{Material and method}

Mössbauer spectra of Martian soil, obtained with the use of MIMOS spectrometers located on Spirit and Opportunity Rovers were the objects of the studies [4].

Mössbauer measurements were performed with the use of a ${ }^{57} \mathrm{Co}$ source the activity of which, at the time of landing, was about $150 \mathrm{mCi}$. The time for each measurement was six to twelve hours. The measurements were continued up to 1000 days after landing.

Measurements of the Martian soil were performed in the backscattering geometry.

Martian Mössbauer spectrometers were also using transmission geometry for collecting calibration measurements. For every measurement of Martian soil, a calibration spectrum was attached. Because of big differences in the temperatures on the surface of Mars, Mössbauer spectra of the same object were divided into spectra obtained at different range of the temperatures (see Fig. 1).

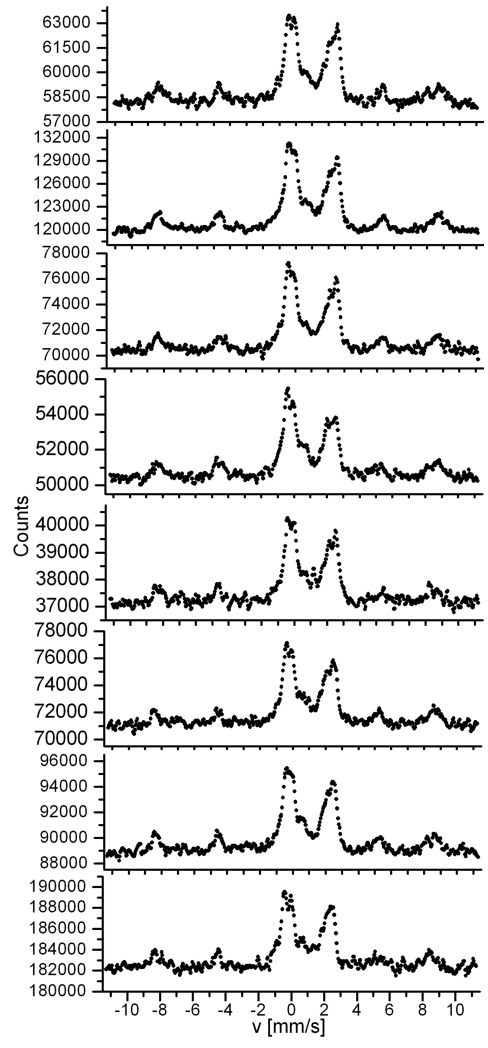

Fig. 1. Mössbauer spectra obtained on the surface of Mars from soil 23 in different temperatures windows, from top to the bottom: $200-210 \mathrm{~K}, 210-220 \mathrm{~K}, 220-$ $230 \mathrm{~K}, 230-240 \mathrm{~K}, 240-250 \mathrm{~K}, 250-260 \mathrm{~K}, 260-270 \mathrm{~K}$, $270-280 \mathrm{~K}$

Data from measurements performed during the mission MER are available in the original form in the Internet on the web side of the Planetary Data System.

To use these original data a special program is needed.

The constructors of the MIMOS spectrometers provided a simple program which reads the original files and writes them in csv format. Data about measurements, calibration and non-linearity of the drive are available. These programs do not calibrate the spectra and are not user friendly. 
The alternative program is the MERView program. The MERView program was written by Professor David Agresti from Alabama University in Birmingham, U.S. This program not only reads the data, but calibrates and folds them. Program MERView eliminates also the nonlinearity of the drive.

Calibrated and folded spectra are ready for analysis by a conventional program for analyzing Mössbauer spectra, e.g. Recoil.

\section{Results and discussion}

To create the catalogue of the Martian Mössbauer spectra we used a program written by David Agresti [5].

In the catalogue there are all the Mössbauer spectra collected by Opportunity, which we were able to reconstruct with the use of the MERView program. The catalogue consists of 815 spectra measured during 153 days (when Mössbauer experiments were being performed). The data were taken from the PDS (Planetary Data System) database created by NASA (12). Each file was transformed into a text file and then calibrated relative to $\alpha$-Fe and folded.

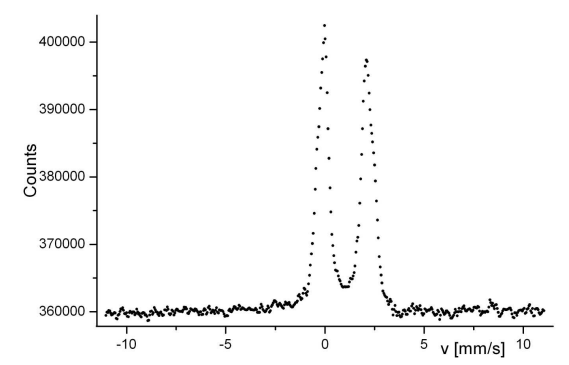

Fig. 2. Typical Mössbaer spectrum of Martian regolith (The Catalogue of Martian Mössbauer Spectra group I).

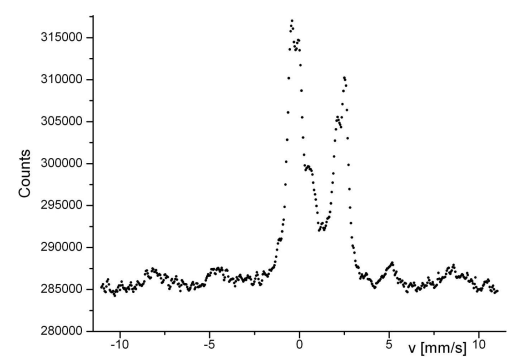

Fig. 3. Typical Mössbaer spectrum of Martian regolith (The Catalogue of Martian Mössbauer Spectra group II)

The spectra were divided into four groups. In first group there are spectra without a magnetic phase and with two maxima. In the second group there are spectra with four maxima and a small magnetic fraction. In the third group there are spectra with a large magnetic phase and with a paramagnetic phase. In the fourth group there are spectra mainly with a magnetic phase.

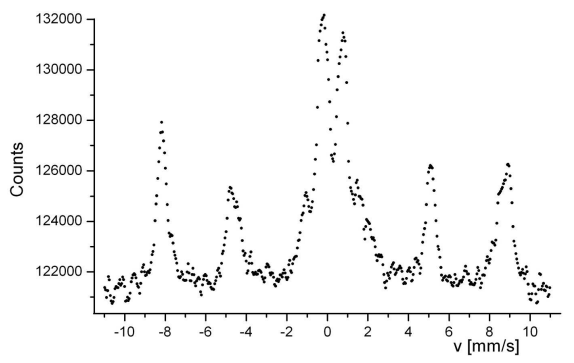

Fig. 4. Typical Mössbaer spectrum of Martian regolith (The Catalogue of Martian Mössbauer Spectra group III).

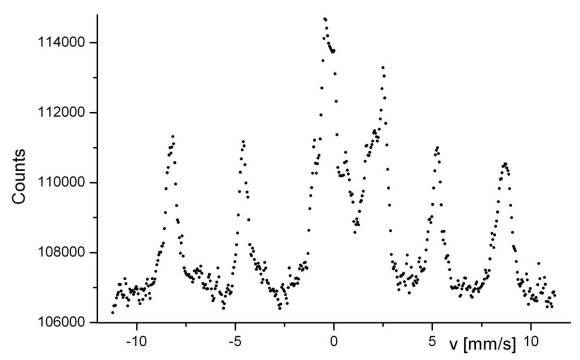

Fig. 5. Typical Mössbaer spectrum of Martian regolith (The Catalogue of Martian Mössbauer Spectra group IV).

In Figs. 2-5 spectra typical for each group are presented. For this presentation the spectra with the best statistics were chosen.

The aim of the construction of this catalogue was to create the possibility to compare quickly the spectra from different places on the Martian surface.

\section{References}

[1] J. Gałązka-Friedman, Postępy Astronomii 36, 233 (1988).

[2] J. Gałązka-Friedman, J. Juchniewicz, Project acepted by Cosmic Research Center in Moscow (February 1989).

[3] G. Klingelhöfer, J. Foh, P. Held, H. Jäger, E. Kankeleit, R. Teucher, Hyperfine Interact. $\mathbf{7 1}$, 1449 (1992).

[4] G. Klingelhöfer, R.V. Morris, P.A. De Souza, D. Rodionov, C. Schröder, Hyperfine Interact. 170, 169 (2006).

[5] D. Agresti, M. Darby Dyar, M.W. Schafer, LPS 37, 1517 (2005). 